\title{
MEASUREMENT OF THE DIELECTRIC PROPERTIES OF PARATELLURITE AT MICROWAVES USING A BICONICAL RESONATOR
}

\author{
M. V. Andreev, O. O. Drobakhin, and D. Yu. Saltykov* \\ Oles Honchar Dnipro National University, Dnipro, Ukraine \\ *e-mail:d.yu.saltykov@gmail.com
}

\begin{abstract}
The results of measurements of the dielectric parameters of $\mathrm{TeO}_{2}$ samples in the microwave range are presented. The measurements were carried out in $X$-band by the cavity perturbation method using a measuring setup based on a biconical resonator. The advantages of using a high-Q biconical resonator for measuring the dielectric parameters of a paratellurite sample are considered. The determination of the dielectric parameters of the samples is based on finite element computational model and using digital method for determining the natural frequency by fractional rational approximation of the frequency response. The influence of the deviation of the sample shape from the cylindrical on the resonator parameters and the measurement error is considered. The estimate of the dielectric constant of $\mathrm{TeO}_{2}$ in the crystallographic direction [110] was 24.4 , the dielectric loss factor such as $\tan \delta$ is estimated as $3 \cdot 10^{-4}$. The results obtained are in good agreement with the reference results presented in the scientific literature.

Keywords: paratellurite, dielectric constant, dielectric loss tangent, biconical resonator, cavity resonator method.

Received 10.10.2020; Received in revised form 21.11.2020; Accepted 02.12.2020
\end{abstract}

\section{Introduction}

For studying the properties of new materials, it is often necessary to measure and test their parameters in various frequency ranges of radio wave radiation. In particular, it is of certain practical interest to test dielectric parameters changing as the result of effect of microwave radiation on the properties of acousto-optical crystals, for example, $\mathrm{TeO}_{2}$, which is a promising active material for creating ultrafast optically switchable devices [1]. To carry out such research, it is necessary to ensure sufficient accuracy and unambiguity with simultaneous non-invasiveness of measurements of the parameters of the test materials.

Various electromagnetic microwave systems with the dependences of their parameters on electromagnetic properties of filling materials are used for constructing the sensors (primary measuring transducers) for the determination of the electrophysical parameters of materials in the microwave range. Microwave measurement methods are non-contact, which makes it possible to carry out research without destroying the samples and without the presence of additional errors associated with contact phenomena. Various resonance systems are widely used as sensors for determining the parameters of dielectrics in microwave measuring devices [2-3].

Cavity resonator method is based on measurements of the values of the scattering matrix $S_{11}$ or $S_{21}$ of resonators with the test sample. Using the measured values, the desired characteristics of the dielectric material are calculated. Cavity perturbation method is applied to investigate materials with both low and high dielectric constant values, with both low and high losses using the design of the cavity resonance frequency or the method of the test sample replacement. The samples should have the simplest possible shape to simplify the subsequent calculation and small geometrical dimensions that do not cause strong distortion of the electromagnetic field of the resonator. The main sources of errors in microwave cavity perturbation method are inaccuracy in measuring the values of $S_{11}$ and $S_{12}$, calculation errors, inaccuracies in the manufacture and determination of the location of the test sample. Supplementing the experimental procedure with a digital method for determining the natural frequency of the resonator based on a fractional rational approximation of the frequency response [4] can significantly improve the accuracy of the results.

\section{Measurement method}

Among the types of resonators used for the material parameter measurements, open 
waveguide resonators with cutoff regions [4-5] occupy a specific place. One of the simplest resonators of this type is the biconical resonator, which is a combination of two truncated cones with a common base [4,6]. The resonator is configured in such a way that cutoff conditions arise in the tapering regions. As a result, high-Q resonant oscillations appear in the resonator due to the re-reflection of electromagnetic waves from the critical sections located in the tapering parts of the resonator.

The cutoff sections allow creating holes for introducing the test samples into the cavity, which practically do not affect the field structure and cavity parameters. In a biconical resonator, the main disadvantages of regular cylindrical structures are eliminated: the need to ensure the perpendicularity of the end walls, a sharp decrease in the Q-factor of the resonator caused by the presence of holes in the end walls of the resonator, and the frequency degeneracy of the $\mathrm{H}_{01 p}$ and $\mathrm{E}_{11 p}$ oscillations. The last effect is especially important, since lower-quality mode $\mathrm{E}_{11 p}$ are excited by any inhomogeneities inside the resonator, in particular, when the test sample disturbs the field, which leads to a decrease in the accuracy and unambiguity of measurements.

There is a lot of papers devoted to the study of dielectric parameters using single and coupled biconical resonators, for example [7]. Fig. 1 shows the simplest sensor based on a biconical resonator for monitoring the parameters of dielectrics. The test sample of cylindrical shape is introduced along the axis of the resonator through the end holes in the sections $z_{1}$ and $z_{2}$ located in the cutoff regions of the resonator. Usually, especially when the parameters of materials with a high dielectric constant are being measured, azimuthally symmetric modes $\mathrm{H}_{01 p}$ are used. A feature of such modes is a high Q-factor $\left(\sim 10^{5}\right)$, which is associated with the absence of longitudinal currents in the resonator walls. The critical sections $z_{c 1}$ and $z_{c 2}$ are located at a considerable distance from the tops of the cones, which makes it possible to create holes for the introduction of test samples with sufficiently large radius of cylinder, almost without resonator characteristics degrading.

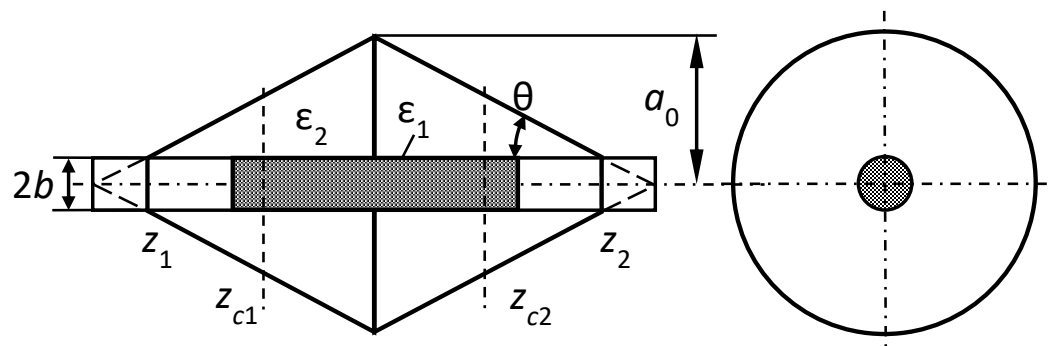

Fig. 1. Axially filled biconical resonator.

When the test sample is introduced into the resonator volume, the structure of the electromagnetic field is disturbed, as a result, the resonant frequency and Q-factor of the resonant system change. The parameters of the test material can be determined from changes in the characteristics of the resonator. To carry out measurements, it is necessary to provide an unambiguous links between the parameters of the resonator and the test sample parameters, therefore it is important to exclude as much as possible the influence of various external factors, such as changes in the temperature and humidity of the environment, instability of the measuring equipment, etc.

Important factors affecting the accuracy and unambiguity of measurements are the deviations of the geometric parameters of the test sample from the specified ones. To improve the measurement accuracy, it is necessary to ensure a high degree of cylindricality of the sample and concentricity of its placement in the resonator. Unfortunately, in practice, it is far from always possible to produce a sample of an ideal 102 
cylindrical shape, particularly, for ceramic and crystalline materials with high hardness and brittleness. In this case, the shape of the samples under study can differ significantly from the cylindrical one.

The results of investigating the influence of deviations of the geometric parameters of the test sample on the characteristics of biconical resonators and on the accuracy of determining the parameters of dielectrics with their help are presented in [8]. Numerical analysis shows that the deviation of the shape of dielectric samples from cylindrical form leads to significant changes in the resonant frequency of a biconical resonator. This effect is enhanced with an increase in the geometric dimensions and permittivity of the samples. For samples with a dielectric constant of $\sim 3.8$ and a radius of $2-3 \mathrm{~mm}$, a deviation from cylindricity by $0.1 \mathrm{~mm}$ leads to a change in frequency by several MHz. In turn, a frequency deviation of $1 \mathrm{MHz}(0.01 \%$ of the resonant frequency) leads to an error in determining the dielectric constant of the order of $10 \%$. To measure the dielectric constant with an error of $\sim 5 \%$, it is necessary to provide the frequency change less than $2.5 \mathrm{MHz}$, and the deviation from cylindricity for the samples under consideration not exceeding $\sim 0.05 \mathrm{~mm}(2-3 \%)$. The deviation of the coaxiality of the test sample in the biconical resonator with the same order of deviations introduces a much smaller error in the measurements of the dielectric constant than the violation of the cylindricity of the sample. It is found that samples with a radius of $2.5-3.0 \mathrm{~mm}$, which is approximately 0.1 of the maximum radius $a_{0}$ of the biconical resonator, are optimal for measurements with the considered biconical resonator. To improve the accuracy of determining the dielectric constant and reduce the effect of deviations from cylindricity, it is advisable to carry out measurements for several samples of different radii. Naturally, when materials with high dielectric constants are studied, the requirements to the quality of fabrication and placement of samples increase significantly.

\section{Experimental results}

The investigation of the properties of the tested acousto-optic $\mathrm{TeO}_{2}$ crystals were carried out in the X-band using a measuring setup based on standard measuring equipment: generator G4-83 (frequency band 7.5 - 10.5 GHz) and spectrum analyzer S427. The biconical resonator with a maximum radius of $a_{0}=25.25 \mathrm{~mm}$ and a half angle at the apex of the cone $\theta=30^{\circ}$, made of copper, was used as a measuring transducer. The end hole radius was $5.5 \mathrm{~mm}$. The resonator was connected to the waveguide path through circular diaphragms with radii of 3.5 and $4.3 \mathrm{~mm}$, located in the plane of the central section of the resonator. The resonant frequency of the empty resonator for the $\mathrm{H}_{011}$ mode was $9515 \mathrm{MHz}$, the loaded Q-factor was 12700, the accuracy of determining the absolute value of the frequency was $\pm 10 \mathrm{MHz}$, and the relative change in frequency was $\pm 0.5 \mathrm{MHz}$.

The test samples were cut from $\mathrm{TeO}_{2}$ crystals obtained by the Czochralski method in the crystallographic direction [110]. The samples deviated from the ideal cylindrical shape; the length of the samples was slightly less than the length of the resonator. For example, the cross-sectional diameter of a paratellurite sample in mutually perpendicular planes was 7.9 and $8.4 \mathrm{~mm}$, and the sample length was $38.6 \mathrm{~mm}$ (the total cavity length was $66.5 \mathrm{~mm}$ ). Thin-walled polyethylene tubes were used to fix the samples along the resonator axis. Depending on the orientation of the "flattened" cylindrical sample in the transverse plane, the resonance frequency of the biconical resonator with the paratellurite sample varied in the range $10231-10243 \mathrm{MHz}$, the loaded Q-factor - in the range 6500 9300.

The determination of the dielectric parameters of the samples was based on a computational model by the finite element method. To reduce the measurement error, the 
dielectric constant was determined from the relative change in the resonance frequency to the frequency $\mathrm{H}_{011}$ mode of the empty resonator, the dielectric loss factor was determined from the relative change in the Q-factor. For the test sample, the dielectric constant was $\varepsilon=24.4 \pm 0.2$, and the dielectric loss tangent was $\tan \delta=(3 \pm 1) \cdot 10^{-4}$.

In some cases, obtaining samples of cylindrical shape is difficult; it is much easier to make the sample in the form of a parallelepiped. When examining rectangular samples, the measurement error associated with the greater dependence of the resonance frequency on the angle of rotation of the sample in the transverse plane of the resonator is compensated by the simplicity of measuring and controlling this angle and, as a result, practically does not affect the accuracy of determining the dielectric constant of the test material. For example, for a rectangular paratellurite sample with dimensions of $7.8 \times 6.2 \times 30 \mathrm{~mm}$, the resonance frequency varied in the range $10175-10183 \mathrm{MHz}$, the measured dielectric constant was $\varepsilon=22 \pm 0.3$.

\section{Conclusions}

The use of biconical resonators makes it possible to measure the parameters of materials with high dielectric constant. Estimates of the dielectric constant and dielectric loss tangent for paratellurite samples obtained by the Czochralski method are in good agreement with those known from the scientific literature, for example [1].

\section{Acknowledgments}

We would like to thank to the staff of the Laboratory of Physics of Crystals of Active Dielectrics of the Oles Honchar Dnipro National University for the provided $\mathrm{TeO}_{2}$ samples.

\section{References}

1. Li, Yanlu. Structural, electronic, and optical properties of $\alpha, \beta$, and $\gamma-\mathrm{TeO}_{2} / \mathrm{Yanlu} \mathrm{Li}$, Weiliu Fan, Honggang Sun, Xiufeng Cheng, Pan Li, Xian Zhao // Journal of Applied Physics. 2010. - Vol. 107, 093506.

2. Egorov, V.N. Resonance methods for microwave studies of dielectrics. (Review) / V.N. Egorov // Instruments and Experimental Techniques. - 2007. - Vol. 50, No. 2. - P. 143-175.

3. Microwave Electronic: Measurement and Materials Characterization / L.F. Chen, C.K. Ong, C.P. Neo, V.V. Varadan, and V.K. Varadan - Chichester, WS, England: John Wiley \& Sons, Ltd., 2004. -537 p.

4. Andreev, M.V. Determination of biconical cavity eigenfrequencies using method of partial intersecting regions and approximation by rational fractions / M.V. Andreev, O.O. Drobakhin, D.Yu. Saltykov, N.B. Gorev, I.F. Kodzhespirova // Radioelectronics and Communications Systems, 2019. - Vol. 62, No. 12. - P. 630-641.

5. Drobakhin, O.O. Open-ended Waveguide Cutoff Resonators for Monitoring Dielectrics Parameters of Gases / O.O. Drobakhin, Ye.N. Privalov, D.Yu. Saltykov // Telecommunications and Radio Engineering. - 2013. - Vol. 72, No 7. - P. 627-640.

6. Andreev, M.V. Simple technique for biconical cavity eigenfrequency determination / M.V. Andreev, O.O. Drobakhin, D.Yu. Saltykov, N.B. Gorev, I.F. Kodzhespirova // Radioelectronics and Communications Systems, 2017. - V. 60, No. 12. - P. 555-561.

7. Andreev, M. V. Measurement of Dielectric Material Properties Using Coupled Biconical Resonators / M. V. Andreev, O. O. Drobakhin, Ye. N. Privalov, D. Yu. Saltykov // Telecommunications and Radio Engineering. - 2014. - Vol. 73, No 11. - P. 1017-1032.

8. Andreev, M. V. The Effect of Cylindricity and Coaxiality Deviations of Samples on Measurement Error of the Permittivity Using a Biconical Resonator / M.V. Andreev, O.O. Drobakhin, D.Yu. Saltykov // Proc. 2019 24th Int. Seminar/Workshop Direct and Inverse Problems of Electromagnetic and Acoustic Wave Theory, Lviv, Ukraine, Sept. 11-14, 2019. - P. 55-58. 